\title{
1 Mutations in MAGEL2 and L1CAM are associated with congenital
}

\section{hypopituitarism and arthrogryposis}

3 Gregory LC ${ }^{1}$, Shah $\mathrm{P}^{2}$, Sanner $\mathrm{JRF}^{2}$, Arancibia $\mathrm{M}^{3}$, Hurst $\mathrm{JA}^{4}$, Jones WD4 ${ }^{4}$, Spoudeas

$4 \quad \mathrm{H}^{2}$, Le Quesne Stabej P1, Williams HJ ${ }^{1}$, Ocaka LA ${ }^{1}$, GOSgene $^{5}$, DDD study ${ }^{6}$, Loureiro

$5 \quad \mathrm{C}^{3}$, Martinez-Aguayo $\mathrm{A}^{3}$, Dattani $\mathrm{MT}^{1,2} .{ }^{1}$ Genetics and Genomic Medicine Programme,

6 UCL Great Ormond Street Institute of Child Health, London, United Kingdom, ${ }^{2}$ Great

7 Ormond Street Hospital, London, United Kingdom, ${ }^{3}$ Division de Pediatria, Escuela de

8 Medicina, Pontificia Universidad Catolica de Chile, ${ }^{4} \mathrm{NE}$ Thames Genetics Service,

9 Great Ormond Street Hospital, London, United Kingdom, ${ }^{5}$ NIHR Biomedical Research

10 Centre at Great Ormond Street Hospital, Children NHS Foundation Trust and UCL,

11 London, United Kingdom. ${ }^{6}$ Deciphering Developmental Disorders, Wellcome Trust

12 Sanger Institute, Wellcome Trust Genome Campus, Cambridge, UK.

Word count: 3227

\section{Short title}

Patients with hypopituitarism and arthrogryposis.

\section{Keywords}

Hypopituitarism; arthrogryposis; Schaaf-Yang syndrome; L1 syndrome; MAGEL2, L1CAM

Corresponding author: Professor Mehul T Dattani MD FRCP FRCPCH Academic Affiliation:

Professor of Paediatric Endocrinology

Genetics and Genomic Medicine Programme

UCL Great Ormond Street Institute of Child Health

30 Guilford Street

London WC1N 1EH

Tel no. 02079052657

Email:m.dattani@ucl.ac.uk 
Congenital hypopituitarism $(\mathrm{CH})$ is rarely observed in combination with severe joint contractures (arthrogryposis). Schaaf-Yang syndrome (SHFYNG) phenotypically overlaps with Prader-Willi syndrome, with patients also manifesting arthrogryposis. L1 syndrome: a group of X-linked disorders including hydrocephalus and lower limb spasticity, also rarely presents with arthrogryposis.

We investigated the molecular basis underlying the combination of $\mathrm{CH}$ and arthrogryposis in five patients. The heterozygous p.Q666fs*47 mutation in the maternally imprinted MAGEL2 gene, previously described in multiple SHFYNG patients, was identified in Patients 1-4, all of whom manifested growth hormone deficiency and variable SHFYNG features, including dysmorphism, developmental delay, sleep apnea and visual problems. Non-identical twins (Patients 2 and 3) had diabetes insipidus and macrocephaly, and Patient 4 presented with $A C T H$ insufficiency. A hemizygous L1CAM variant, p.G452R, previously implicated in L1 syndrome patients, was identified in Patient 5, who presented with antenatal hydrocephalus.

Human embryonic expression analysis revealed MAGEL2 transcripts in the developing hypothalamus and ventral diencephalon at Carnegie stages (CS) 19, 20 and 23, and in Rathke's pouch at CS20 and 23. L1CAM was expressed in the developing hypothalamus, ventral diencephalon and hindbrain (CS19, 20, 23), but not in Rathke's pouch.

We report MAGEL2 and $\angle 1 C A M$ mutations in four pedigrees with variable $\mathrm{CH}$ and 
be examined for features of SHFYNG and/or L1 syndrome. This study highlights the association of hypothalamo-pituitary disease with MAGEL2 and L1CAM mutations.

\section{Introduction}

Schaaf-Yang syndrome (SHFYNG) (OMIM: 615547) is a rare congenital disorder that is often mis-diagnosed as Prader-Willi syndrome (PWS) (OMIM: 176270), but includes arthrogryposis within the phenotypic spectrum. Arthrogryposis multiplex congenita (OMIM: 208100), commonly known as arthrogryposis, occurs in 1/3000 live births and involves multiple congenital joint contractures in two or more areas of the body, resulting from reduced or absent fetal movement. Arthrogryposis multiplex congenita has been reported in a patient with pituitary ectopia, who had seizures thought to be caused by hypoglycemia and who was later found to have a small anterior and an ectopic posterior pituitary (PP); however, no genetic etiology was identified (1). The main overlapping characteristic features of SHFYNG and PWS are hypotonia, feeding difficulties during infancy, global developmental delay/intellectual disability and sleep apnea (2-4). Patients with SHFYNG, however, lack certain stereotypical PWS features such as hyperphagia and subsequent obesity. PWS is linked to a specific locus $15 q 11$ q13 within the genome, where five maternally imprinted (paternally expressed) genes, namely MKRN3, MAGEL2, NDN, NPAP1, SNURF-SNRPN, and six maternallyimprinted small nucleolar RNA (snoRNA) genes/clusters are located (3). Different deletions in this region give rise to variable PWS with a combination of genes being responsible for different manifestations of the disease (5-7).

L1 syndrome describes a range of $\mathrm{X}$-linked disorders including spastic paraplegia, MASA (Mental retardation, Aphasia, Spasticity, and Adducted thumbs) syndrome 
82 (OMIM: 303350), X-linked hydrocephalus with stenosis of the aqueduct of Sylvius (HSAS) (OMIM: 307000), and X-linked complicated corpus callosum agenesis (8). L1 syndrome occurs in 1/30,000 individuals and includes hydrocephalus, variably severe intellectual deficit, and spasticity of the lower limbs, with generalized contractures in rare cases. MASA syndrome, named after the characteristic phenotypes present in patients, also includes adducted thumbs in $50 \%$ of cases. A small number of patients (<20) have a combination of L1 syndrome and Hirschsprung disease, a rare disorder affecting the colon leading to severe constipation and intestinal obstruction due to missing ganglion cells in the myenteric (Auerbach's) plexus in the colon (9).

In this study, we sought to investigate the genetic etiology in five patients from four unrelated families who presented with variable congenital hypopituitarism $(\mathrm{CH})$ and arthrogryposis.

\section{Materials and Methods}

\section{Exome sequencing of Patients 1-5}

The full coding region of Patients 1-5 were sequenced by GOSgene, London UK (Patients 1 and 5), GOSH UK as part of the Deciphering Developmental Disorders (DDD) Study (Patients 2 and 3) and by colleagues at the Pontificia Universidad Catolica de Chile (Patient 4). Raw sequencing data were mapped against the GRCh37/hg18 reference genome and data were analyzed using the Ingenuity® Variant Analysis ${ }^{\mathrm{TM}} \quad$ software (https://www.qiagenbioinformatics.com/ products/ingenuity-variant-analysis) from QIAGEN, Inc (GOSgene). All remaining filtered variants were considered to be potentially pathogenic disease-causing 
mutations. Exome sequencing and data analysis for Patients 1 and 5 were performed by GOSgene as previously described (10), for Patients $2-3$ under the DDD study as previously described (11), and for Patient 4 by Ambry Genetics (www.ambrygen.com) using their standard protocol and filtering criteria. Mutations were confirmed in the patients via Sanger sequencing using specifically designed exon-spanning primers that amplify the DNA region containing the variant (annealing temperatures and primer sequences are available upon request). A chromosome microarray was also performed on the twins (Patients 2-3) (specific details of this protocol are available upon request). The appropriate ethical approval for the genetics and human embryonic tissue expression studies has been obtained prior to this project taking place. The patients/patient guardians gave full consent to all clinical and genetic studies carried out on their blood/DNA.

\section{Human embryonic expression studies using in situ hybridisation}

Human embryonic tissue sections were obtained from the Human Developmental Biology tissue Resource (HDBR) (http://hdbr.org) and selected from Carnegie stage (CS) 16, 19, 20 and 23 (equivalent to gestational age (GA) 5.5, 6, 7 and 8 weeks) respectively. Digoxigenin (DIG) RNA probes were made using purified vectors containing the full-length human cDNA of wild-type MAGEL2 (in the pCR4-TOPO vector, IMAGE ID: 8327725) and L1CAM (in the pCR-XL-TOPO vector, IMAGE ID: 8991945) (Source Bioscience) respectively. Gene expression studies were performed by in situ hybridisation as previously described (12), to generate a human embryonic hypothalamo-pituitary expression profile for both MAGEL2 and L1CAM. 
131 Results

132 Patient 1

133 A white European patient presented at the age of 3.2 years with short stature, 134 hypoglycemia, and arthrogryposis with scoliosis and a flexion deformity of the knees. 135 She was hypotonic since birth and required nasal oxygen until 5 weeks of age. A 136 respiratory collapse at 7 weeks of age necessitated a prolonged PICU admission. She 137 was also noted to have laryngeal polyps. She was diagnosed with growth hormone 138 deficiency (GHD), with a peak $\mathrm{GH}$ of $6.4 \mu \mathrm{g} / \mathrm{L}$ and an undetectable IGF-I, at age 3.7 139 years (Table 1). GH treatment was commenced at 4 years of age (Figure 1A). 140 Dysmorphic features were noted, including bulbar palsy, a long face, a prominent 141 forehead and micrognathia. She also had global developmental delay and a squint 142 with mild optic nerve hypoplasia $(\mathrm{ONH})$ and cerebral visual impairment. She had central sleep apnea and gastro-esophageal reflux. MRI of the brain was reported normal (Figure 2A).

\section{Patients 2 and 3}

146 Female non-identical white European twins with distal arthrogryposis were initially 147 referred with hypernatremia, and were then diagnosed with diabetes insipidus (DI) shortly after birth. Subsequent short stature led to a diagnosis of GHD [peak GH to stimulation of $4.8 \mu \mathrm{g} / \mathrm{L}$ and $3.2 \mu \mathrm{g} / \mathrm{L}$ respectively, with an undetectable IGF-I, at $0.8 \mathrm{y}$; (Table 1)]. Their DI was treated with Desmopressin since birth and $\mathrm{GH}$ treatment commenced after 1 year of age (Figure 1B-C). The patients had distinctive features 
152 including macrocephaly, a long face with bi-temporal narrowing, frontal bossing, scaphocephaly, micrognathia and a cleft/high arched palate. Patient 2 had nystagmus with optic nerve atrophy and was severely sight impaired, whilst her sister had $\mathrm{ONH}$ with visual impairment. They both had global developmental delay. Patient 2 is wheelchair bound and unable to speak, whilst Patient 3 is able to stand and has basic vocalization. The twins also had central sleep apnea and scoliosis. Patient 2 had chronic lung disease with supplemental oxygen requirement at night and had a tracheostomy until the age of 6 years. Patient 3 had a tracheostomy until 20 months of age. On MRI, Patient 2 showed evidence of progressive global cerebral hemisphere atrophy with relative preservation of the posterior fossa structures, with a thin corpus callosum, a small PP, and optic nerve hypoplasia (Figure 2A). Patient 3 had generalised underdevelopment of the brain with a mature right parieto-occipital infarct and a thin corpus callosum, optic nerve hypoplasia, and a normal PP (Figure 2B).

\section{Patient 4}

A male Caucasian patient from Chile presented with short stature and a deceleration in growth rate at the age of 2.8 years. He was diagnosed with GHD (a stimulation test latter consisted of contractures, shortening of the extremities, and limited extension of the elbows, knees, hips, and fingers, namely camptodactyly. He was started on hydrocortisone at 2.9 years and GH treatment at 3.5 years of age (Figure 1D). He had strabismus, global developmental delay with autism spectrum disorder (ASD), generalized hypotonia and dysmorphic features including a long face with bi-temporal narrowing, a prominent forehead, micrognathia, glossoptosis and a high arched 
palate. He had gastroesophageal reflux and central sleep apnea, with respiratory

177

178 complications leading to a tracheostomy. Cardiac complications included an osteum secundum interauricular communication with spontaneous closure. Cryptorchidism resolved with a bilateral orchidopexy. His MRI was normal (Figure 2D).

\section{$\underline{\text { Patient } 5}$}

A male Afro-Caribbean patient presented with antenatal ventriculomegaly and dysmorphic features including bilateral radial clubbed hands and plagiocephaly. Flexion deformities that affected both the wrists and hands were noted antenatally, and he was diagnosed with distal arthrogryposis with adducted thumbs and flexion deformities of his digits post-natally. A ventriculo-peritoneal shunt was inserted at 4 days of age, and hypoglycemic seizures ensued at the age of 0.7 years. He was later diagnosed with GHD (peak GH 3.7 $\mu \mathrm{g} / \mathrm{L}$; undetectable IGF-I) and GH treatment was commenced from 1 year of age (Table 1) (Figure 1E). Gastrointestinal problems included dysphagia, and the patient was fed via a percutaneous endoscopic gastrostomy. Other phenotypic features present in this patient included a ventricular septal defect, severe obstructive sleep apnea, global developmental delay, generalised hypotonia, right hip subluxation and scoliosis. Bilateral astigmatism with a left divergent squint and subsequent visual impairment were apparent upon eye examination. His MRI revealed a bulky tectum, generalised white matter loss and a thin corpus callosum, with no evidence of obstructive hydrocephalus (Figure 2C). 
200 Whole exome sequencing was performed on the 5 patients with $\mathrm{CH}$ and arthrogryposis 201 at three different institutions respectively. The results identified the MAGEL2 c.1996dupC, p.Q666Pfs*47 truncation mutation in Patient 1 (GOSgene), Patients 2-3

203

204

205

206

207

208 (GOSH UK as part of the Deciphering Developmental Disorders (DDD) Study), and Patient 4 (Pontificia Universidad Catolica de Chile). A chromosome microarray was also performed on the twins (Patients 2-3), which revealed a 16q11 duplication, 45,186,600-45,416,670, in Patient 2 only. A hemizygous L1CAM C.1354G>A, p.G452R variant was identified in Patient 5 (GOSgene) who also had hydrocephalus and other features consistent with L1 syndrome. The p.G452R variant is located at a highly conserved residue across multiple species and is located within the lg5 extracellular domain of the L1 protein. Both MAGEL2 p.Q666Pfs*47 and L1CAM p.G452R are absent from control databases, including the gnomAD browser (http://gnomad.broadinstitute.org/).

\section{Human embryonic expression profile of MAGEL2 and L1CAM using in situ} hybridisation

\section{MAGEL2}

At the early embryonic stage of CS16, there is no MAGEL2 expression in the developing hypothalamus or in Rathke's pouch (RP) (the primordium of the anterior pituitary). However, there is strong transcript staining specifically in the inferior ganglion of the vagus nerve and the spinal ganglia. At CS19, MAGEL2 mRNA transcripts appear in the hypothalamus and the spinal cord, but are undetectable in 
222 RP. At CS20, strong expression is present throughout the ventral diencephalon, and 223 in both RP and the PP. This expression is maintained within the hypothalamus and $224 \mathrm{RP}$ at CS23 and noted in the trigeminal ganglia (Figure 3). There was no staining 225 visualised using the sense control probe on equivalent sections at any stage.

\section{$\underline{L 1 C A M}$}

228

There was no L1CAM mRNA transcript staining at CS16 in the human embryonic brain sections incorporating the hypothalamus and RP. At CS19 there is strong expression in the hypothalamus and trigeminal ganglia, but not in RP. Staining was also noted in the metencephalon and throughout the ventral diencephalon at this stage. L1CAM expression is maintained in the hypothalamus and forebrain as well as the hindbrain during CS20 and 23 (Figure 4). No staining was observed in RP or in the PP at any stage analysed in this study. The sense control probe produced no staining at any stage.

\section{Discussion}

MAGEL2 is a member of the type II MAGE gene family involved in neurogenesis and brain function $(13,14)$. It is thought to enhance ubiquitin ligase activity $(15)$, act as a regulator of retrograde transport and promote endosomal F-actin assembly, and is involved in the regulation of the circadian clock (16). In humans, loss of function point mutations causing truncations in the MAGEL2 gene were initially implicated in the etiology of variable PWS-like features and contractures of the small finger joints, a phenotype now commonly referred to as SHFYNG syndrome (3). 
Magel2-null mice present with similar features to PWS in humans, including neonatal growth retardation, excessive weight gain after weaning, impaired hypothalamic regulation, reduced fertility and excess fat with decreased muscle mass (17-20). Additionally, Magel2-knock out mice elicit altered social phenotypes and impaired ability to distinguish between known and novel partners (21). Recent studies have concluded that POMC neuron activity and its communication with downstream targets is significantly compromised (22), and that oxytocin neuronal activity is suppressed (23) in Magel2-deficient mice.

Specific association of the MAGEL2 gene with PWS was first suggested following expression studies using northern blotting, where MAGEL2 was expressed in the adult human brain, notably the hypothalamus, and in the fetal brain (however details were not specific), lung and kidney (24). The authors concluded that loss of MAGEL2 may explain abnormalities in brain development in PWS individuals. Expression analysis performed in the current study has further characterised the location of MAGEL2 transcripts within the developing fetal human brain. We have shown that MAGEL2 is highly expressed in the developing hypothalamus from 6 to at least 8 weeks GA, and in the developing pituitary gland (RP) at 7-8 weeks GA (Figure 3), supporting the hypothesis that this gene plays a critical role during embryonic brain development.

The MAGEL2 mutation c.1996dupC, p.Q666Pfs*47 identified in Patients 1-4 has been previously identified in two siblings diagnosed with a neurodevelopmental disorder including hypotonia, ASD, hyperinsulinemic hypoglycemia and features of arthrogryposis (25). Subsequently, the c.1996de/C, causing a frameshift in the same location, p.Q666Sfs*36, was described in three patients with a lethal form of arthrogryposis (26). Both the c.1996delC deletion and c.1996dupC duplication have 
since been identified in multiple SHFYNG patients. These data widened the

270 phenotypic spectrum of SHFYNG, expanding the range to include fetal akinesia and arthrogryposis $(27,28)$. In previous reports of patients harboring MAGEL2 truncating mutations, intellectual disability varied from mild to severe, and ASD was not always present. The majority of affected patients had arthrogryposis (varying in severity), short stature, and hypogonadism, which are all common features in SHFYNG patients $(3,27,28)$, with one female patient manifesting hypogonadotropic hypogonadism $(\mathrm{HH})$ (27). Interestingly, a recent report describes the first SHFYNG patient with early onset obesity to harbor a MAGEL2 truncation (de novo c.1850G>A, p.Trp617*) (29).

GHD has frequently been identified in SHFYNG patients; however other pituitary deficits have not been described until recently. Two siblings and an unrelated female patient with SHFYNG, arthrogryposis and severe respiratory difficulties were found to carry truncating MAGEL2 variants, p.Q638* and p.S1044* respectively, and manifested variable hypopituitarism (30). One of the siblings was diagnosed with central diabetes insipidus and gonadotrophin deficiency, whilst the unrelated patient was diagnosed with panhypopituitarism including GHD, central hypothyroidism, adrenal insufficiency, and gonadotrophin deficiency, with a hypoplastic anterior pituitary gland on MRI (30). Patients 2 and 3 from the current study manifest DI, and Patient 4 has multiple pituitary hormone deficiency including GHD and ACTH insufficiency. This is the first association of the p.Q666Pfs 47 frameshift with endocrinopathies in SHFYNG patients. Together with the previous report (30), these findings further highlight how different MAGEL2 truncations seem to play a role in the etiology of both DI and CPHD as part of SHFYNG syndrome, which until recently were not major phenotypic features reported in such patients. Another recent case report has identified the novel MAGEL2 p.Q1007* truncation in a SHFYNG patient with GHD, 
hypothyroidism and hyperprolactinaemia (31), again suggesting that variable $\mathrm{CH}$ is being increasingly identified in these patients. Interestingly, a previous report described a patient with Moebius syndrome, GHD and arthrogryposis (32). Although no genetic mutations were identified in this patient, it demonstrates the link between these diverse phenotypes.

A recent publication reported the first association of MAGEL2 truncation mutations with Chitayat-Hall syndrome (OMIM: 208080), which has a strong phenotypic overlap with SHFYNG (33). Chitayat-Hall syndrome is characterized by distal arthrogryposis, intellectual disability, dysmorphic features and hypopituitarism, with GHD being present in all reported cases to date (34). The same p.Q666Pfs*47 MAGEL2 truncation was present in one of the Chitayat-Hall syndrome patients reported, demonstrating how variable overlapping phenotypes between SHFYNG and ChitayatHall syndrome arise from the same genotype, and suggesting that full length MAGEL2 is crucial for normal development of the human brain, and for normal hypothalamopituitary function. Chitayat-Hall syndrome and SHFYNG may in fact be the same syndrome albeit with variable penetrance, with some patients having sleep apnea, currently noted as a characteristic feature of SHFYNG but not Chitayat-Hall. There are an increasing number of patients with SHFYNG with MAGEL2 mutations (35) that have not had their hypothalamo-pituitary function tested, suggesting that pituitary dysfunction may be a more frequent feature of SHFYNG, as is observed with ChitayatHall syndrome. Early endocrine diagnosis is crucial if endocrine morbidity is to be prevented, and therefore essential for improvement of the quality of life of these complex patients. 
Mutations in L1CAM, located on the X chromosome (Xp28) and encoding the L1 protein, have been implicated in the etiology of L1 syndrome (8). Female carriers may also manifest minor features of this syndrome such as adducted thumbs or mild intellectual deficit (36). L1 is an axonal glycoprotein cell adhesion molecule that plays a role in neuronal migration and differentiation, including axon fasciculation (37), neurite outgrowth (38), synapse formation (39) and myelination (40). L1CAM-null mice have hydrocephalus, a smaller hippocampus and cerebellum, corpus callosal hypoplasia, hyperfasciculation of the corticothalamic tracts, and pyramidal tract abnormalities (41-46). Mutations within the cytoplasmic domain of the L1 protein (L1CD) have been described in MASA syndrome, which led to murine studies with L1CD disruption. Surprisingly these mice have normal brain morphology, although they have defects in motor function (47). The hemizygous L1CAM mutation, p.G452R, identified in Patient 5 has been described previously in a patient with severe hydrocephalus (48). This mutation lies within, and is predicted to affect, the structure of the L1 extracellular domain required for correct folding of the protein, and subsequently thought to affect binding through the distortion of domain conformation (49). Further investigations supported this, with a decreased ligand-binding ability in the presence of $L 1 C A M$ p.G452R (50).

In rodents, L1cam is expressed in migrating neuron cell bodies from embryonic stage 9.5 and is later expressed in growing and regenerating axons. Myelinating Schwann cells express L1CAM during embryonic and postnatal development, whilst nonmyelinating Schwann cells express L1CAM through adulthood (51-54). The human L1CAM expression profile generated in this study revealed high transcript expression in the hypothalamus from 6-8 weeks of development (Figure 4). However, no expression was visible in RP or the PP, suggesting that this gene is hypothalamic and 
plays a critical role in this region during brain development. Patient 5 is the first patient to our knowledge that has an L1CAM mutation and manifests GHD with pituitary dysfunction associated with features of L1 syndrome.

The trigeminal ganglia are sensory ganglia of the trigeminal nerve, responsible for sensation in the face and for motor functions. Both MAGEL2 and L1CAM expression within these specific tissues and during midline craniofacial development may suggest that the sensation in the face may be impaired in patients with mutations in these genes. However, the presence of global developmental delay did not allow assessment of this function. Limited availability of human embryonic sections did not allow analysis of expression beyond 8 weeks of gestation.

To summarise, our data suggest that patients with SHFYNG and L1 syndromes should all be screened and monitored for hypothalamo-pituitary abnormalities. Furthermore, $\mathrm{CH}$ patients with accompanying joint contractures should be screened for MAGEL2 and $L 1 C A M$ mutations and evaluated/monitored for additional phenotypes commonly present in SHFYNG or L1 syndrome respectively. Our data and previously published data on SHFYNG and L1 syndromes suggest that MAGEL2 or L1CAM, respectively, should be screened for mutations using Sanger sequencing before next generation techniques are conducted, as there is a high chance that a mutation lies within these genes in such patients. This would be the most cost-effective approach in screening for the most likely genetic diagnosis. However, in those cases where a mutation is not identified in either of these genes, either whole exome or genome sequencing may be performed. 
Figure 1 (A-E): Growth charts of (A) Patient 1, (B) Patient 2 and (C) Patient 3, (D)

\section{Patient 4 and (E) Patient 5.}

The red labelled arrow indicates when growth hormone (GH) treatment commenced in the patients respectively. The purple arrow on (D) indicates commencement of hydrocortisone.

Figure 2: Magnetic resonance imaging (MRI) for Patients 2, 3 and 5.

(A) MRI of Patient 1. MRI shows a normal anterior and posterior pituitary, with no other anomalies. (B) MRI of Patient 2 (twin). MRI reveals global cerebral hemisphere atrophy with a small posterior pituitary, a thin corpus callosum and small optic nerves. (C) MRI of Patient 3 (twin). MRI reveals generalised underdevelopment of the brain. The posterior pituitary was normal with small optic nerves and a thin corpus callosum. (D) MRI of Patient 4. MRI shows a normal anterior and posterior pituitary, with no other anomalies. (E) MRI of Patient 5. MRI shows generalised underdevelopment of the brain and a very thin corpus callosum. AP, anterior pituitary; PP, posterior pituitary; WML, white matter loss; CC, corpus callosum; ON, optic nerve.

Figure 3: Human MAGEL2 expression during embryonic development.

(A) Carnegie stage (CS) 16, the equivalent of 5.5 weeks into embryonic development. MAGEL2 expression is noted in the inferior ganglion of the vagus (IGV) nerve and the spinal ganglia (SG). (B) At CS19, 6 weeks into development, there are high levels of mRNA transcripts in the developing hypothalamus (Hyp), ventral diencephalon (VD), and (C) spinal cord (SC). (D) At CS20, 7 weeks into development, strong transcript staining is present throughout the VD, and in both Rathke's pouch (RP) and the 
posterior pituitary (PP). (E) A magnified image of the RP and PP from image (D). (F)

At CS23, 8 weeks into development, MAGEL2 expression is maintained in the Hyp, $\mathrm{RP}$ and $\mathrm{PP}$, with some expression in the trigeminal ganglia (TG).

(A-B) A human embryonic section from Carnegie stage (CS) 19 showing L1CAM mRNA transcripts in the developing hypothalamus (Hyp), ventral diencephalon (VD) and trigeminal ganglia (TG). (B) mRNA transcripts can be seen in the spinal cord (S).

\section{Acknowledgments}

Great Ormond Street Hospital (GOSH) charity and the Medical Research Foundation (MRF) (grant\# 535963) funded this study. The human embryonic and fetal material was provided by the Joint MRC/Wellcome Trust (grant\# MR/R006237/1) Human Developmental Biology Resource (http://hdbr.org). The research in this study is in part supported by the NIHR, GOSH and BRC. The views expressed are those of the 
003], a parallel funding partnership between Wellcome and the Department of Health,

and the Wellcome Sanger Institute [grant number WT098051]. The views expressed

in this publication are those of the author(s) and not necessarily those of Wellcome or

Republic of Ireland REC). The research team acknowledges the support of the

National Institute for Health Research, through the Comprehensive Clinical Research

Network.

1. Parano E, Trifiletti RR, Barone R, Pavone V, Pavone P. Arthrogryposis multiplex congenita and pituitary ectopia. A case report. Neuropediatrics. 2000;31(6):325-7. Willi syndrome: consensus diagnostic criteria. Pediatrics. 1993;91(2):398-402. mutations of MAGEL2 cause Prader-Willi phenotypes and autism. Nature genetics. 2013;45(11):14058.

4. Fountain MD, Schaaf CP. Prader-Willi Syndrome and Schaaf-Yang Syndrome: Neurodevelopmental Diseases Intersecting at the MAGEL2 Gene. Diseases (Basel, Switzerland). 2016;4(1).

5. Sahoo T, del Gaudio D, German JR, Shinawi M, Peters SU, Person RE, et al. Prader-Willi phenotype caused by paternal deficiency for the HBII-85 C/D box small nucleolar RNA cluster. Nature genetics. 2008;40(6):719-21.

6. de Smith AJ, Purmann C, Walters RG, Ellis RJ, Holder SE, Van Haelst MM, et al. A deletion of the HBII-85 class of small nucleolar RNAs (snoRNAs) is associated with hyperphagia, obesity and hypogonadism. Human molecular genetics. 2009;18(17):3257-65.

7. Duker AL, Ballif BC, Bawle EV, Person RE, Mahadevan S, Alliman S, et al. Paternally inherited microdeletion at 15q11.2 confirms a significant role for the SNORD116 C/D box snoRNA cluster in Prader-Willi syndrome. European journal of human genetics : EJHG. 2010;18(11):1196-201. 8. Kanemura Y, Okamoto N, Sakamoto H, Shofuda T, Kamiguchi H, Yamasaki M. Molecular mechanisms and neuroimaging criteria for severe L1 syndrome with X-linked hydrocephalus. Journal of neurosurgery. 2006;105(5 Suppl):403-12.

9. Okamoto N, Wada Y, Goto M. Hydrocephalus and Hirschsprung's disease in a patient with a mutation of L1CAM. Journal of medical genetics. 1997;34(8):670-1.

10. Reid ES, Williams H, Stabej Ple Q, James C, Ocaka L, Bacchelli C, et al. Seizures Due to a KCNQ2 Mutation: Treatment with Vitamin B6. JIMD reports. 2016;27:79-84. 

post-hoc comparison of the utility of sanger sequencing and exome sequencing for the diagnosis of heterogeneous diseases. Human mutation. 2013;34(12):1721-6.

453 12. Gregory LC. Investigation of new candidate genes in a cohort of patients with familial congenital hypopituitarism and associated disorders. [Doctoral Thesis]: UCL (University College London); 2017.

13. Bischof JM, Ekker M, Wevrick R. A MAGE/NDN-like gene in zebrafish. Developmental dynamics : an official publication of the American Association of Anatomists. 2003;228(3):475-9.

14. Lopez-Sanchez N, Gonzalez-Fernandez Z, Niinobe M, Yoshikawa K, Frade JM. Single mage gene in the chicken genome encodes CMage, a protein with functional similarities to mammalian type II Mage proteins. Physiological genomics. 2007;30(2):156-71.

15. Doyle JM, Gao J, Wang J, Yang M, Potts PR. MAGE-RING protein complexes comprise a family of E3 ubiquitin ligases. Molecular cell. 2010;39(6):963-74.

16. Tacer KF, Potts PR. Cellular and disease functions of the Prader-Willi Syndrome gene MAGEL2. The Biochemical journal. 2017;474(13):2177-90.

17. Bischof JM, Stewart CL, Wevrick R. Inactivation of the mouse Magel2 gene results in growth abnormalities similar to Prader-Willi syndrome. Human molecular genetics. 2007;16(22):2713-9.

18. Mercer RE, Wevrick R. Loss of magel2, a candidate gene for features of Prader-Willi syndrome, impairs reproductive function in mice. PloS one. 2009;4(1):e4291.

19. Tennese AA, Wevrick R. Impaired hypothalamic regulation of endocrine function and delayed counterregulatory response to hypoglycemia in Magel2-null mice. Endocrinology. 2011;152(3):96778 .

20. Kamaludin AA, Smolarchuk C, Bischof JM, Eggert R, Greer JJ, Ren J, et al. Muscle dysfunction caused by loss of Magel2 in a mouse model of Prader-Willi and Schaaf-Yang syndromes. Human molecular genetics. 2016;25(17):3798-809.

21. Fountain MD, Tao H, Chen CA, Yin J, Schaaf CP. Magel2 knockout mice manifest altered social phenotypes and a deficit in preference for social novelty. Genes, brain, and behavior. 2017;16(6):592-600.

22. Oncul M, Dilsiz P, Ates Oz E, Ates T, Aklan I, Celik E, et al. Impaired melanocortin pathway function in Prader-Willi syndrome gene-Magel2 deficient mice. Human molecular genetics. 2018;27(18):3129-36.

23. Ates T, Oncul M, Dilsiz P, Topcu IC, Civas CC, Alp MI, et al. Inactivation of Magel2 suppresses oxytocin neurons through synaptic excitation-inhibition imbalance. Neurobiology of disease. 2019; 121:58-64.

24. Lee S, Kozlov S, Hernandez L, Chamberlain SJ, Brannan CI, Stewart CL, et al. Expression and imprinting of MAGEL2 suggest a role in Prader-willi syndrome and the homologous murine imprinting phenotype. Human molecular genetics. 2000;9(12):1813-9.

25. Soden SE, Saunders CJ, Willig LK, Farrow EG, Smith LD, Petrikin JE, et al. Effectiveness of exome and genome sequencing guided by acuity of illness for diagnosis of neurodevelopmental disorders. Science translational medicine. 2014;6(265):265ra168.

26. Mejlachowicz D, Nolent F, Maluenda J, Ranjatoelina-Randrianaivo H, Giuliano F, Gut I, et al. Truncating Mutations of MAGEL2, a Gene within the Prader-Willi Locus, Are Responsible for Severe Arthrogryposis. American journal of human genetics. 2015;97(4):616-20.

27. Fountain MD, Aten E, Cho MT, Juusola J, Walkiewicz MA, Ray JW, et al. The phenotypic spectrum of Schaaf-Yang syndrome: 18 new affected individuals from 14 families. Genetics in medicine : official journal of the American College of Medical Genetics. 2017;19(1):45-52.

28. McCarthy J, Lupo PJ, Kovar E, Rech M, Bostwick B, Scott D, et al. Schaaf-Yang syndrome overview: Report of 78 individuals. American journal of medical genetics Part A. 2018.

29. Kleinendorst L, Pi Castan G, Caro-Llopis A, Boon EMJ, van Haelst MM. The role of obesity in the fatal outcome of Schaaf-Yang syndrome: Early onset morbid obesity in a patient with a MAGEL2 mutation. American journal of medical genetics Part A. 2018.

30. Enya T, Okamoto N, Iba Y, Miyazawa T, Okada M, Ida S, et al. Three patients with SchaafYang syndrome exhibiting arthrogryposis and endocrinological abnormalities. American journal of medical genetics Part A. 2018;176(3):707-11. 
31. A DH-S, Del Carmen DeMingo-Alemany M, Moreno-Macian F, Rosello M, Orellana C, Martinez F, et al. A Novel Mutation of MAGEL2 in a Patient with Schaaf-Yang Syndrome and

32. Petrus M, Rhabbour M, Clouzeau J, Bat P, Bildstein G, Ibanez MH, et al. [Association of Moebius syndrome and hypopituitarism due to a midline anomaly. A case report]. Annales de pediatrie. 1993;40(6):376-8.

33. Jobling R, Stavropoulos DJ, Marshall CR, Cytrynbaum C, Axford MM, Londero V, et al. Chitayat-Hall and Schaaf-Yang syndromes:a common aetiology: expanding the phenotype of MAGEL2-related disorders. Journal of medical genetics. 2018;55(5):316-21.

34. Chitayat D, Hall JG, Couch RM, Phang MS, Baldwin VJ. Syndrome of mental retardation, facial anomalies, hypopituitarism, and distal arthrogryposis in sibs. American journal of medical genetics. 1990;37(1):65-70.

35. McCarthy J, Lupo PJ, Kovar E, Rech M, Bostwick B, Scott D, et al. Schaaf-Yang syndrome overview: Report of 78 individuals. American journal of medical genetics Part A. 2018;176(12):256474.

36. Kaepernick L, Legius E, Higgins J, Kapur S. Clinical aspects of the MASA syndrome in a large family, including expressing females. Clinical genetics. 1994;45(4):181-5.

37. Stallcup WB, Beasley L. Involvement of the nerve growth factor-inducible large external glycoprotein (NILE) in neurite fasciculation in primary cultures of rat brain. Proceedings of the National Academy of Sciences of the United States of America. 1985;82(4):1276-80.

38. Lagenaur C, Lemmon V. An L1-like molecule, the 8D9 antigen, is a potent substrate for neurite extension. Proceedings of the National Academy of Sciences of the United States of America. 1987;84(21):7753-7.

39. Godenschwege TA, Kristiansen LV, Uthaman SB, Hortsch M, Murphey RK. A conserved role for Drosophila Neuroglian and human L1-CAM in central-synapse formation. Current biology : CB. 2006;16(1):12-23.

40. Barbin G, Aigrot MS, Charles P, Foucher A, Grumet M, Schachner M, et al. Axonal celladhesion molecule L1 in CNS myelination. Neuron glia biology. 2004;1(1):65-72.

41. Ohyama K, Tan-Takeuchi K, Kutsche M, Schachner M, Uyemura K, Kawamura K. Neural cell adhesion molecule L1 is required for fasciculation and routing of thalamocortical fibres and corticothalamic fibres. Neuroscience research. 2004;48(4):471-5.

42. Cohen NR, Taylor JS, Scott LB, Guillery RW, Soriano P, Furley AJ. Errors in corticospinal axon guidance in mice lacking the neural cell adhesion molecule L1. Current biology : CB. 1998;8(1):26-33.

43. Dahme M, Bartsch U, Martini R, Anliker B, Schachner M, Mantei N. Disruption of the mouse L1 gene leads to malformations of the nervous system. Nature genetics. 1997;17(3):346-9.

44. Demyanenko GP, Shibata Y, Maness PF. Altered distribution of dopaminergic neurons in the brain of L1 null mice. Brain research Developmental brain research. 2001;126(1):21-30.

45. Rolf B, Kutsche M, Bartsch U. Severe hydrocephalus in L1-deficient mice. Brain research. 2001;891(1-2):247-52.

46. Wiencken-Barger AE, Mavity-Hudson J, Bartsch U, Schachner M, Casagrande VA. The role of L1 in axon pathfinding and fasciculation. Cerebral cortex (New York, NY : 1991). 2004;14(2):12131.

47. Nakamura Y, Lee S, Haddox CL, Weaver EJ, Lemmon VP. Role of the cytoplasmic domain of the L1 cell adhesion molecule in brain development. The Journal of comparative neurology. 2010;518(7):1113-32.

48. Jouet M, Rosenthal A, Armstrong G, MacFarlane J, Stevenson R, Paterson J, et al. X-linked spastic paraplegia (SPG1), MASA syndrome and X-linked hydrocephalus result from mutations in the L1 gene. Nature genetics. 1994;7(3):402-7.

49. Bateman A, Jouet M, MacFarlane J, Du JS, Kenwrick S, Chothia C. Outline structure of the human L1 cell adhesion molecule and the sites where mutations cause neurological disorders. The EMBO journal. 1996;15(22):6050-9.

50. De Angelis E, MacFarlane J, Du JS, Yeo G, Hicks R, Rathjen FG, et al. Pathological missense mutations of neural cell adhesion molecule L1 affect homophilic and heterophilic binding activities. The EMBO journal. 1999;18(17):4744-53. 
51. Guenard V, Gwynn LA, Wood PM. Transforming growth factor-beta blocks myelination but not ensheathment of axons by Schwann cells in vitro. The Journal of neuroscience : the official journal of the Society for Neuroscience. 1995;15(1 Pt 1):419-28.

52. Akopians A, Runyan SA, Phelps PE. Expression of L1 decreases during postnatal development of rat spinal cord. The Journal of comparative neurology. 2003;467(3):375-88.

53. Martini R, Schachner M. Immunoelectron microscopic localization of neural cell adhesion molecules (L1, N-CAM, and MAG) and their shared carbohydrate epitope and myelin basic protein in developing sciatic nerve. The Journal of cell biology. 1986;103(6 Pt 1):2439-48.

54. Martini R, Schachner M. Immunoelectron microscopic localization of neural cell adhesion molecules (L1, N-CAM, and myelin-associated glycoprotein) in regenerating adult mouse sciatic nerve. The Journal of cell biology. 1988;106(5):1735-46.

55. Gubkina O, Cremer H, Rougon G. Mutation in the neural cell adhesion molecule interferes with the differentiation of anterior pituitary secretory cells. Neuroendocrinology. 2001;74(5):335-46.

56. Trouillas J, Daniel L, Guigard MP, Tong S, Gouvernet J, Jouanneau E, et al. Polysialylated neural cell adhesion molecules expressed in human pituitary tumors and related to extrasellar invasion. Journal of neurosurgery. 2003;98(5):1084-93. 